Review Article

\title{
Effect of Combination of Chinese Herbal Medicine versus Western Medicine on Mortality in Patients after Cardiopulmonary Resuscitation: A Systematic Review and Meta-Analysis of Randomized Controlled Trials
}

\author{
Wenxiu Guo, ${ }^{1}$ Xiaoguang Lu, ${ }^{2}$ Dalong Wang, ${ }^{1}$ Tuo Chen, ${ }^{1}$ Zhiwei Fan, ${ }^{2}$ and Yi Song ${ }^{2}$ \\ ${ }^{1}$ Dalian Medical University, Dalian 116044, China \\ ${ }^{2}$ Department of Emergency Medicine, Affiliated Zhongshan Hospital of Dalian University, Dalian 116001, China
}

Correspondence should be addressed to Xiaoguang Lu; dllxg@126.com

Received 15 September 2015; Revised 15 December 2015; Accepted 24 December 2015

Academic Editor: Arndt Büssing

Copyright (C) 2016 Wenxiu Guo et al. This is an open access article distributed under the Creative Commons Attribution License, which permits unrestricted use, distribution, and reproduction in any medium, provided the original work is properly cited.

Introduction. Although Chinese herbal medicine (CHM) treatment combined with conventional western therapy has been widely used and reported in many clinical trials in China, there is uncertainty about the efficacy of this combination in the treatment of patients after cardiopulmonary resuscitation (CPR). This systematic review aimed to assess whether the risk of mortality has decreased comparing the combination of CHM treatment with conventional western therapy. Methods. To identify relevant studies, the literature search was conducted in Medline, Embase, the Cochrane Library, CBM, CNKI, VIP, and Wanfang database. We included all randomized controlled trials (RCTs) that compared outcomes of patients after CPR taking combination of CHM treatment with those taking just conventional western therapy. Results. This meta-analysis showed that patients randomly assigned to combined CHM treatment group had a statistically significant $23 \%$ reduction in mortality compared with those randomly assigned to conventional western therapy group (RR: 0.77; 95\% CI: 0.70-0.84). Conclusions. This meta-analysis provides evidence suggesting that a combined CHM therapy is associated with a decreased risk of mortality compared with conventional western therapy in patients after CPR. Further studies are needed to provide more evidence to prove or refute our conclusion and identify reasons for the reduction of mortality.

\section{Introduction}

Significant progress has been made during the past half century on improving rate of return of spontaneous circulation (ROSC) by means of modern cardiopulmonary resuscitation (CPR). Nonetheless, mortality of resuscitated patients remains unacceptably high, with population-based studies continuing to report rates of $61 \%-90 \%$ [1-3].

Reasons for the high mortality rate of patients who initially achieve ROSC after experiencing cardiac arrest can be attributed to a unique and complex combination of pathophysiological processes involving multiple organs [4]. Although ischemia of the whole body initially causes global tissue and organ injury, the critical additional damage on multiple organ systems occurs during and after reperfusion
$[5,6]$. The features of pathophysiology after ROSC are often superimposed by the disease or injury that caused the cardiac arrest as well as underlying complications, especially systemic inflammatory response syndrome (SIRS) and multiple organ failure (MODS). Therapies that act on individual organs may aggravate other injured organ systems. The severity of the disease is also associated with the cause of cardiac arrest, the severity of the ischemic insult, and the patient's physical condition which will vary with these factors.

At present, the main conventional western treatments include intensive care unit (ICU) management, early hemodynamic optimization, oxygenation, ventilation, circulatory support, management of acute coronary syndrome (ACS), and other persistent precipitating pathologies. Although there are many treatments, there is still lack of substantial 
progress in decreasing the mortality for patients after CPR. Therefore, an adjunctive or a combined approach should be found to solve this urgent problem.

In traditional Chinese medicine (TCM), the pathophysiological status of patients after CPR was first seen in the description of the Chinese classic medical book Inner Classic of the Yellow Emperor (475 BC-221 BC). It belongs to the category of "Jue-Tuo syndrome" with the main clinical characteristics of pale/dim complexion, cyanosis, cold limbs, profuse perspiration, haziness or dysphoria, weak breathing and pulse, even hyperpyrexia and coma, and so forth. In recent years, CHM treatment combined with conventional western therapy (combined CHM therapy) has been widely used to treat patients after CPR. A large number of clinical trials [7-14] have proved that the approach of combined CHM therapy could decrease mortality and improve prognosis. This systematic review aims to summarize the current research results on the effectiveness of combined CHM therapy on the risk of mortality and the complication rate in patients after CPR.

\section{Materials and Methods}

2.1. Search Strategy and Selection Criteria. A systematic review was conducted in accordance with PRISMA (Preferred Reporting Items for Systematic Reviews and MetaAnalyses) guidelines [15]. The research question, search strategy, inclusion criteria, and statistical analyses were prespecified. The literature search, data extraction, and quality assessment were done independently by two reviewers (DL Wang and WX Guo). Relevant studies were identified by systematic searches in Medline, Embase, the Cochrane Library, CBM, CNKI, VIP, and Wanfang databases from the date of inception until May, 2015. The searching terms were "Cardiac Arrest", "Heart Arrest", "Cardiopulmonary Resuscitation", "advanced cardiac life support", "post-resuscitation or postcardiac arrest/post-cardiac arrest syndrome" and "traditional Chinese medicine", "integrative medicine", "alternativemedicine", and "Chinese herbal medicine". Additionally, reference lists of identified trials and review articles were screened for further relevant publications. There were no restrictions for language, publication date, or publication status.

All completed RCTs that assessed the effects of combined CHM therapy versus conventional western therapy in adult patients after ROSC were eligible for inclusion.

In addition, studies had to investigate all-cause mortality since it was the primary outcome. The secondary outcome was the complication rate. Duplicates were identified and deleted. Reviews, animal studies, case reports, editorials, letters, comments, and conference abstracts were excluded.

2.2. Data Extraction. The obtained articles were evaluated by two reviewers (WX Guo and DL Wang) in accordance with a preconfigured form. For each study, the following variables were extracted: study design, authors, date of publication, patients' characteristics including age, gender, and quantity, Chinese herbal treatment protocol, control intervention, and outcome parameters, length/time of follow-up. Disagreements between reviewers were resolved by a third reviewer (Xiaoguang Lu).

2.3. Quality Assessment. The risk of bias was analyzed using the assessment tool from the Cochrane Handbook [16], which included seven domains: random sequence generation, allocation concealment, blinding of participants and personnel, blinding of outcome assessment, incomplete outcome data, selective reporting, and other sources of bias. "L," "U," and " $H$ " were used as a key for the judgement of each domain, with "low" (L) indicating a low risk of bias, "unclear" (U) indicating that the risk of bias was unclear, and "high" $(\mathrm{H})$ indicating a high risk of bias.

2.4. Data Synthesis and Analysis. We used Review Manager (RM 5.2), provided by the Cochrane Collaborations for data analysis. For dichotomous data, risk ratio (RR) with $95 \%$ confidence interval (CI) was used to present the treatment effect. For continuous data, we presented the treatment effect as the mean difference (MD) with 95\% CI. Fixed-effects models and/or random-effects models were used to calculate $\mathrm{MD}$ and/or RR based on the heterogeneity. Heterogeneity was identified by visual inspection of the forest plots and assessed by using a standard Chi-squared test with a significance level of $P<0.1$. At the same time, heterogeneity was examined with $I^{2}$, where $I^{2}$ values over $50 \%$ indicate a substantial level of heterogeneity [16] and diversity [17]. We used the funnel plot to evaluate the potential publication bias.

\section{Results}

3.1. Study Search and Selection. We yielded a total of 825 publications through electronic searches. After 196 duplicates discarded, we excluded publications based on titles and/or abstracts, mainly because they are reviews, animal studies, case reports, editorials, letters, comments, and conference abstracts. 115 full-text studies were identified for further assessment. Subsequently, studies were excluded in line with the principle of PICOS (Patients-Intervention-ComparisonOutcome-Study Style). Of these, we excluded 100 studies which are mainly irrelevant to the PICOS: the characteristics of patients (18 studies), intervention (14 studies), comparison (16 studies), outcome (11 studies), study style (36 studies), studies where data could not be extracted (3 studies), and duplicate reports (2 studies). Accordingly, 15 studies fulfilled the inclusion criteria and were included in this systematic review. For a summary of the study selection process see Figure 1.

3.2. Description of the Studies. Table 1 summarizes the characteristics of the 15 included trials which were all carried out in China and published in Chinese journals. All trials were RCTs. There were a total of 1066 participants over all included studies. Sample sizes varied from 40 to 250 , with an average of 49.82 participants per trial. With the exception of two studies $[18,19]$, all other included studies reported the average age of the participants. The year of publication ranged from 2004 to 2014. 12 trials reported combined CHM therapy versus conventional western therapy, 2 trials $[20,21]$ reported 


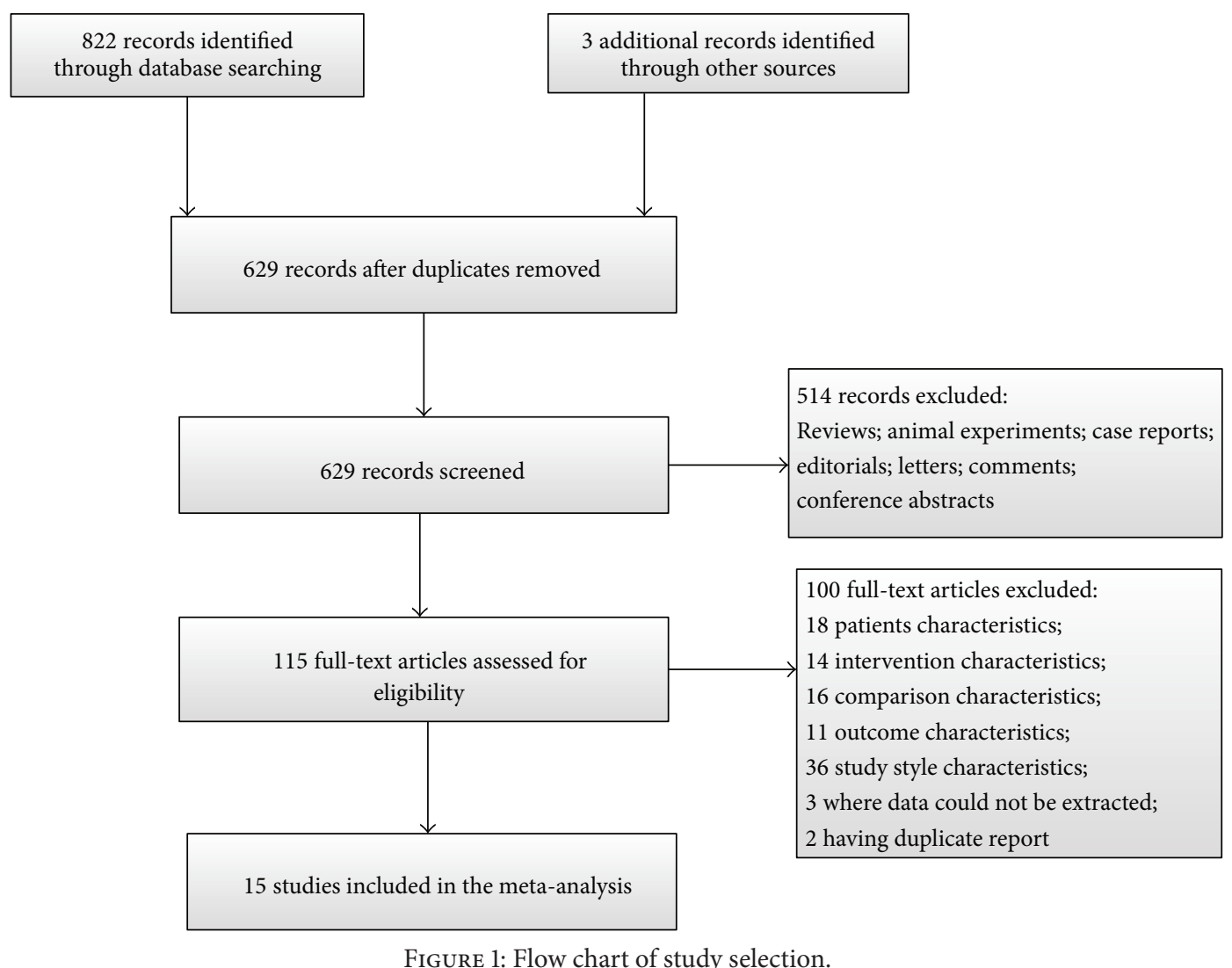

combined CHM therapy versus conventional western therapy plus another western medicine, and 1 trial [19] reported combined CHM therapy versus conventional western therapy plus placebo. For the application of CHM in the combined CHM therapy group, 8 studies $[19,20,22-27]$ reported the use of Shenfu injection (extracted from Renshen and Fuzi), 2 studies [21, 28] reported the use of Shengmai injection (extracted from Renshen, Maidong, and Wuweizi), 2 studies $[29,30]$ reported the use of Xuebijing injection (extracted from Honghua, Chishao, Chuanxiong, Danshen, and Danggui), 1 study [18] reported the use of Erhuang powder (composed of Daihuang and Huangqi), 1 study [31] reported the use of Sini decoction (composed of Ganjiang, Fuzi, and Zhigancao), and 1 study [32] reported the use of Xuefuzhuyujiawei decoction (composed of Renshen, Fuzi, Honghua, Chishao, Chuanxiong, Danggui, Huangqi, Taoren, Shengdihuang, Niuxi, Jiegeng, Chaihu, Zhiqiao, and Gancao). There was one multicentre trial [22]. The duration of the treatment ranged from 3 days to 14 days. With regard to the outcome measures, all trials [18-32] reported the mortality. Seven studies $[18,20-22,27-29]$ reported the complication rate; among them 5 studies $[20-22,27,28]$ reported the incidence of arrhythmia, 1 study [18] reported the incidence of stress-induced gastrointestinal mucosal lesions and toxic enteroparalysis, and 1 study [29] reported incidence of postresuscitation syndrome (PRS).

3.3. Methodological Quality of Included Studies. The risk of bias of included trials was summarized in Figures 2 and
3. According to the Cochrane Collaboration criteria, all of the included trials were evaluated as unclear methodological quality. 15 included trials were described as RCTs. Only 4 trials $[24,25,30,31]$ described the random method for generating random sequences. There was no description on the method of allocation concealment in any of the trials. Only one study mentioned blinding and placebo-controlled method [19]. The number of people at follow-up and the reasons for the failures at follow-up were not reported in any of the trials. There were no withdrawals or dropouts in any of our included trials and all trials reported complete clinical outcome data. The characteristics of participants in different treatment groups of studies were similar and comparable at baseline (gender, age, disease severity, etc.).

\subsection{Effects of Interventions}

3.4.1. The All-Cause Mortality. 15 studies reported the allcause mortality, with a total sample of 1066 patients (544 in the combined CHM therapy group and 522 in the CWT group) [18-31]. A fixed-effects model was used to analyze the data according to the minimal heterogeneity $(P=0.20$; $\left.I^{2}=23 \%\right)$. The meta-analysis of these studies showed a significantly decreased risk of mortality for patients after CPR treated with combined CHM therapy compared with patients treated with conventional western therapy $(\mathrm{CWT})$, with a decrease of 23\% (RR: 0.77; 95\% CI: 0.70-0.84; $P<0.00001$ ) (Figure 4). 


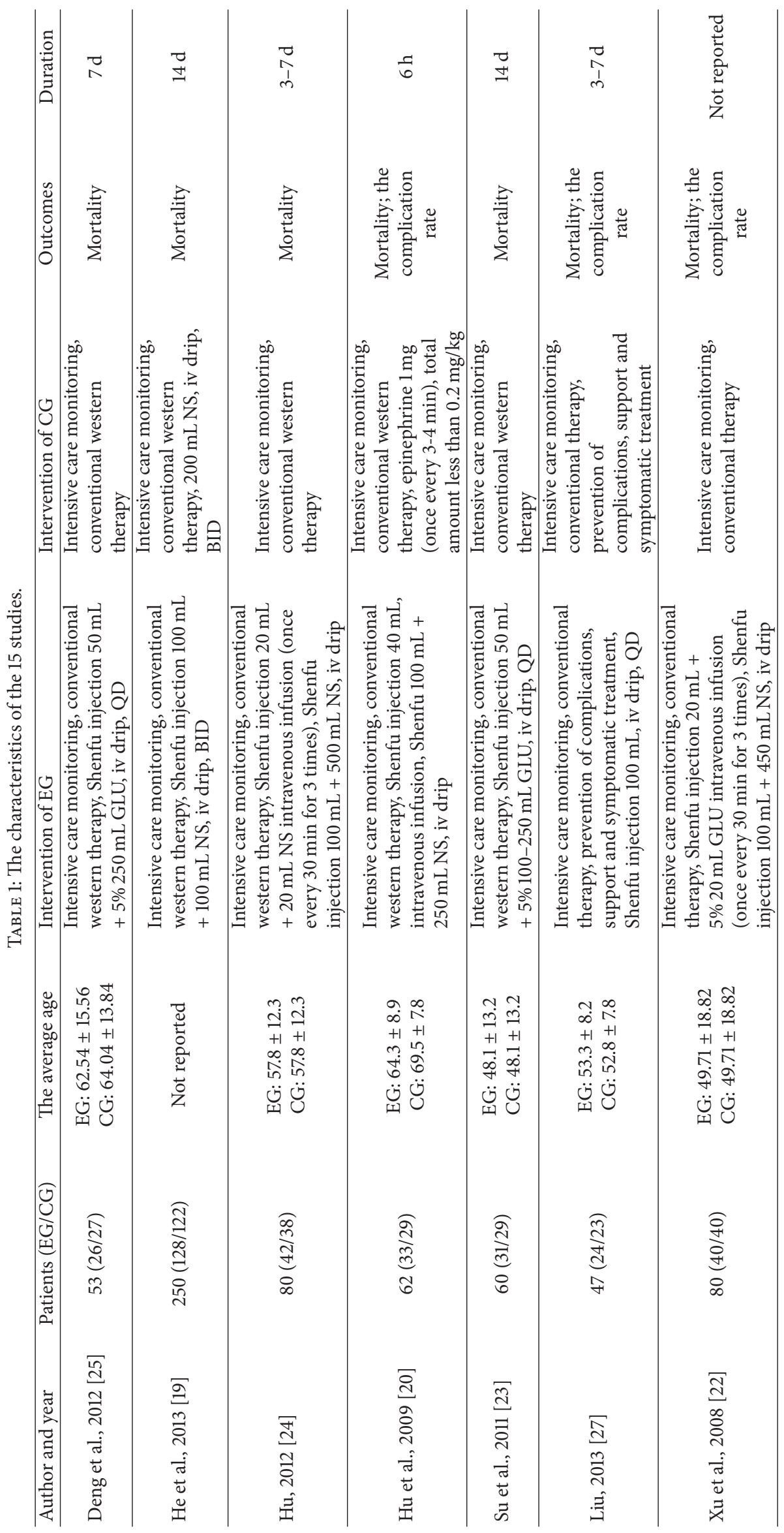



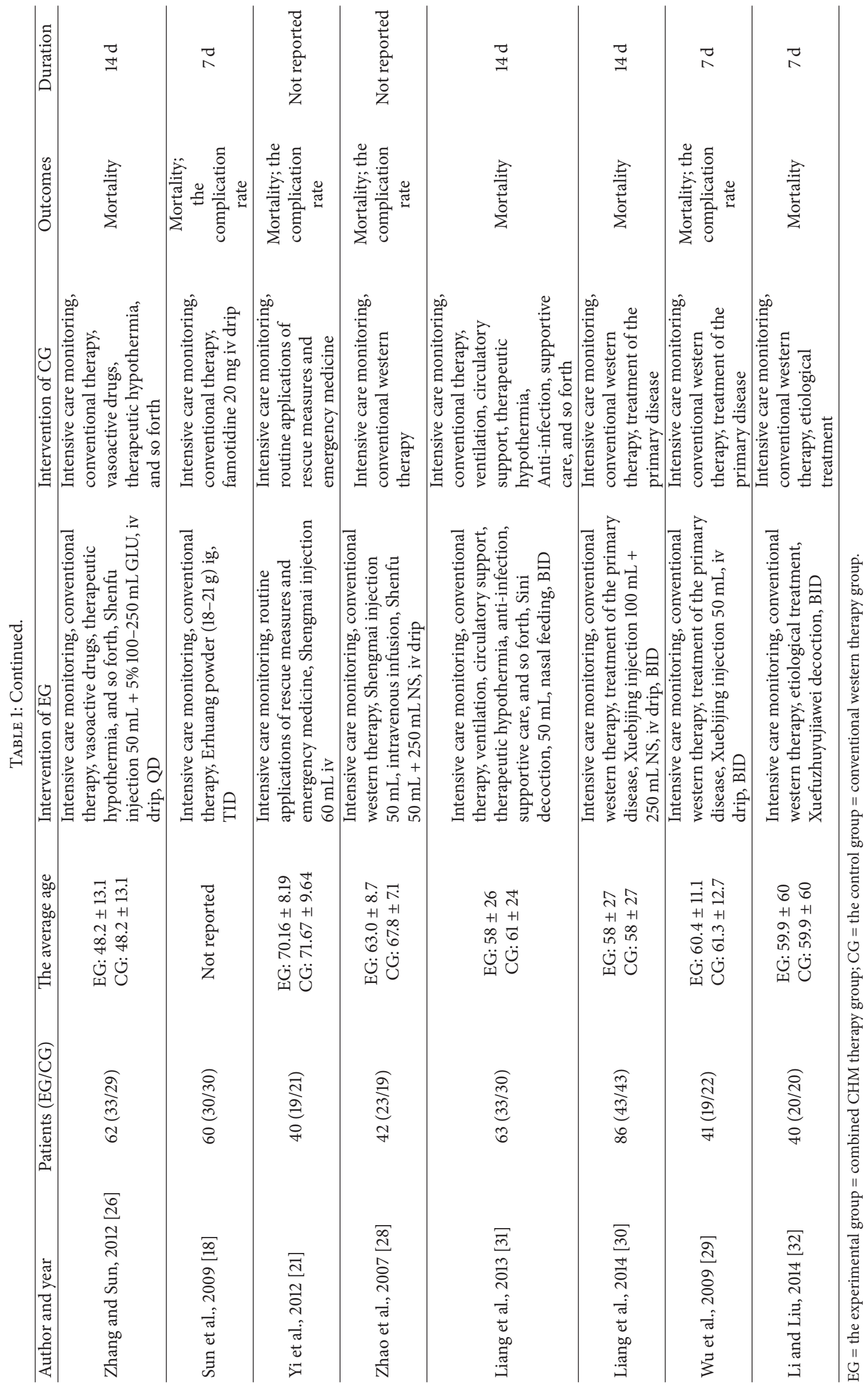


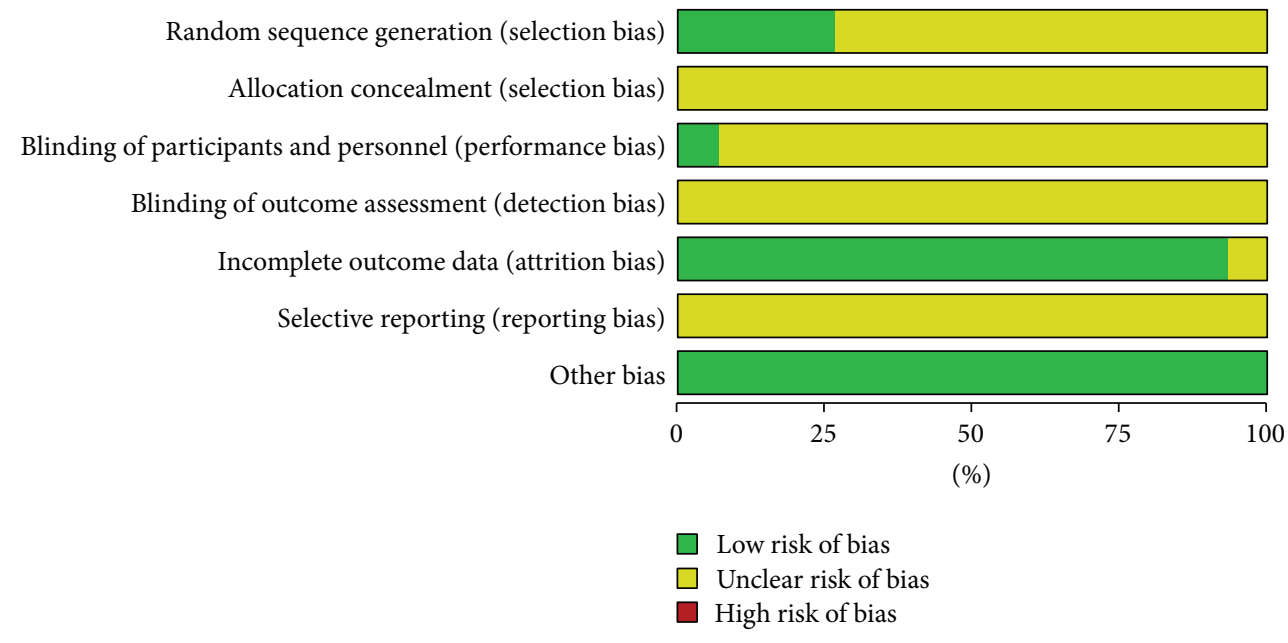

FIGURE 2: Methodological quality graph: review authors' judgements about each methodological quality item presented as percentages across all included studies.

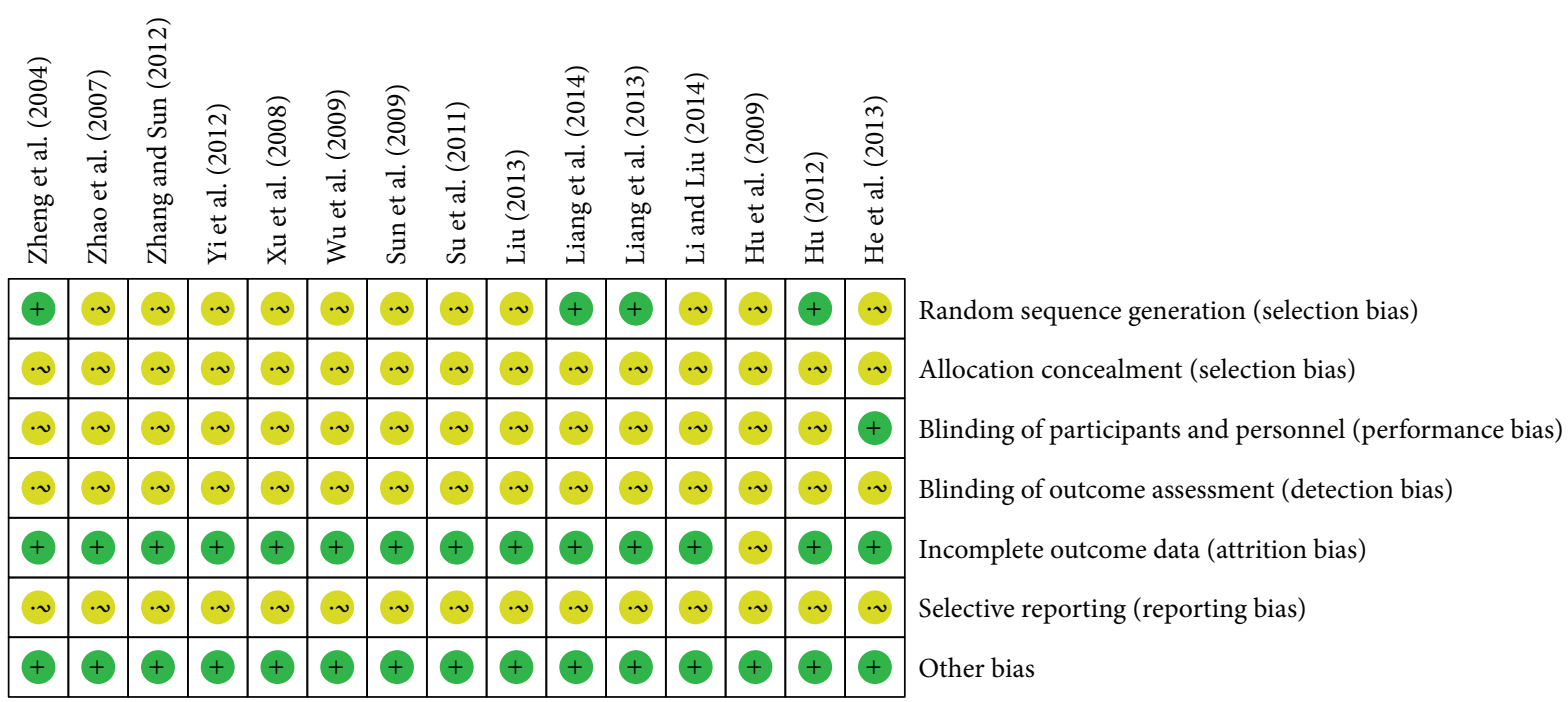

FIGURE 3: Methodological quality summary: review authors' judgements about each methodological quality item for each included study. +: $\mathrm{L}$ (low risk of bias); ?: U (unclear risk of bias); -: H (high risk of bias).

3.4.2. The Complication Rate. Seven studies reported the complication rate, giving a total sample of 372 participants (188 in the combined CHM therapy group and 184 in the CWT group). The data were analyzed using a fixed-effects model according to the heterogeneity test result $(P=$ $\left.0.42, I^{2}=1 \%\right)$. There was significant difference between combined CHM therapy group and conventional western therapy (CWT) group with the RR of 0.64 (95\% CI: 0.56-0.73; $P<0.00001$ ) (Figure 5).

3.4.3. Publication Bias. Because no sufficient number of studies reported the complication rate, we failed to perform funnel plot to detect publication bias on this outcome. Figure 6 was the funnel plot based on studies with data on the all-cause mortality. Results showed that all points in the funnel plots were asymmetrical, indicating that publication bias may have existed in our study.

\section{Discussions}

The high mortality rate after CPR is an urgent problem to be resolved. Currently, the combined CHM therapy has been widely used in China, and a growing number of RCTs have reported that the combined CHM therapy is beneficial for patients after CPR in reducing mortality and improving prognosis. However, there was no systematic review or metaanalysis evaluating the therapeutic effect of combined CHM therapy. So, we conducted this meta-analysis.

Our systematic review of 15 RCTs included 1066 patients and showed a $23 \%$ reduction in all-cause mortality (RR: 0.77; 95\% CI 0.70-0.84; $P<0.00001$ ) in patients receiving combined CHM therapy compared with those assigned to conventional western therapy group. Secondly, combined CHM therapy was associated with significantly lower rates of complications (RR: 0.64; 95\% CI: 0.56-0.73; $P<0.00001$ ), 


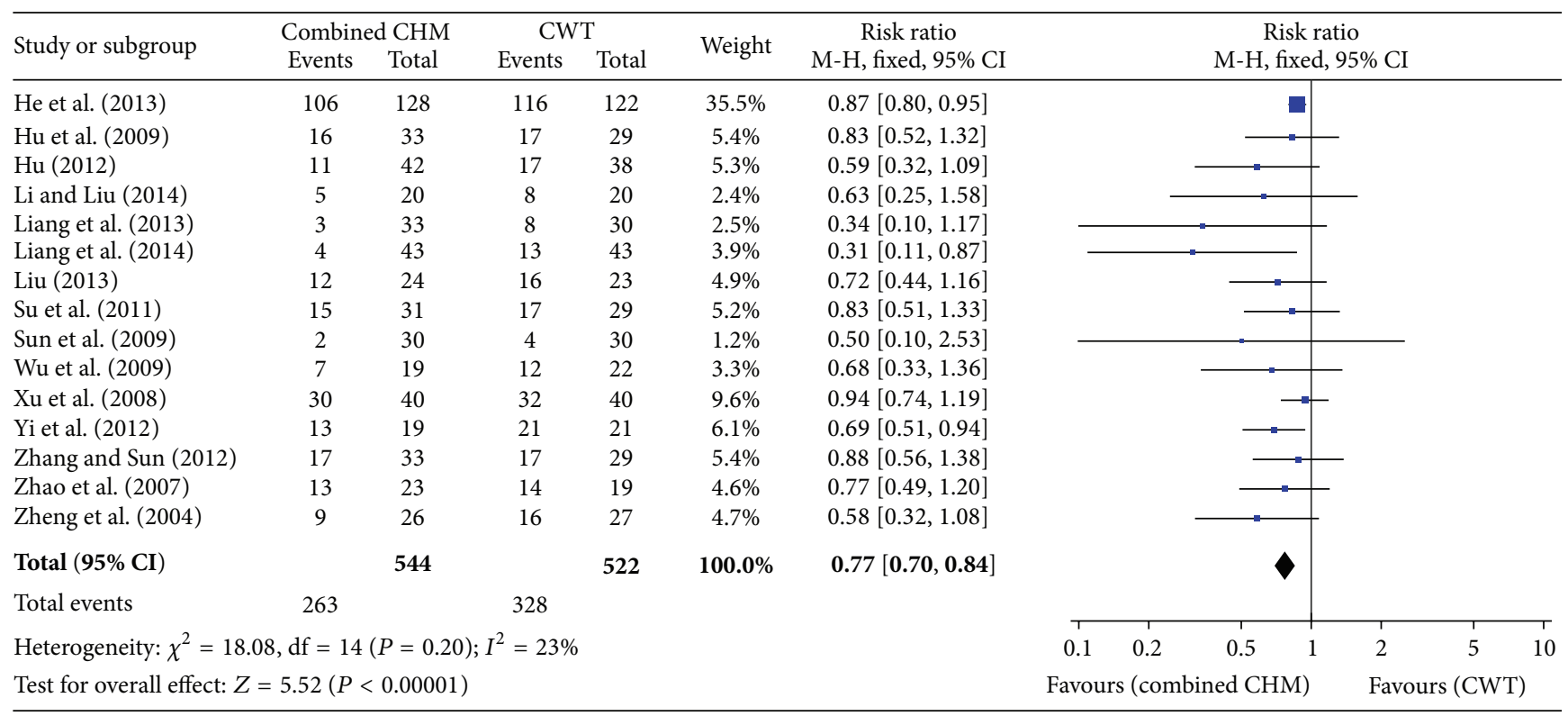

FIGURE 4: Forest plot of all-cause mortality.

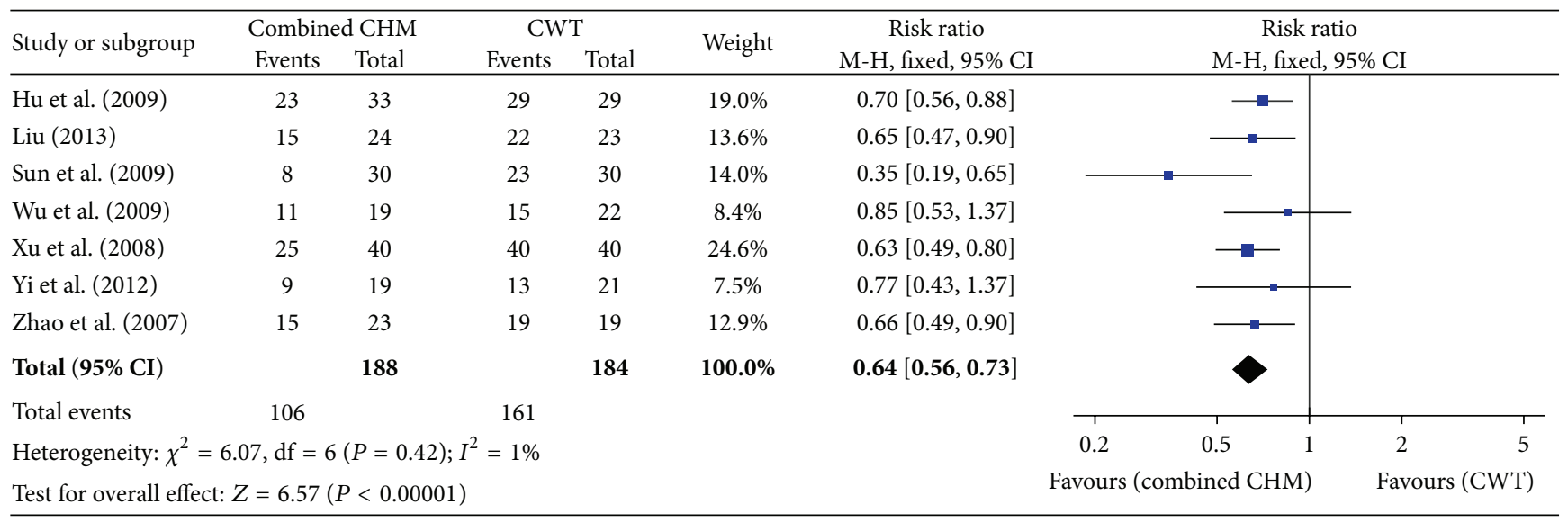

Figure 5: Forest plot of the complication rate.

which further confirmed the effectiveness of the combined CHM therapy in reducing the all-cause mortality. However, because of fewer studies we included in this endpoint and potential reporting bias existing, we should be careful to draw the conclusion that the reason for decreasing in mortality is that the combined CHM therapy could lower the complication rate. Further studies are needed to confirm our viewpoint and identify reasons for the reduction of mortality.

It is a very unique and complex pathophysiological process for patients after CPR, involving multiple organs. The whole-body ischemia-reperfusion injury is the mainly pathological mechanism of patients after CPR and at the same time the complications (such as MODS and SIRS) caused by it are the most important cause of high mortality. The conventional western medicine treatments are mainly concentrated in the treatment of primary disease leading to cardiac arrest and preventing those complications. However, almost all of resuscitated patients are faced with excessive consumption of physical function, as well as organs in an overloaded state, or it could be understood that there was an excessive destruction of the body's defense capabilities. This is the most critical starting point of CHM treatmentreplenishing the consumption of physical function, improving defense capabilities of the whole body and resistance to disease. In traditional Chinese medicine (TCM), the main treatments are supplementing Qi, warming Yang, nourishing Yin, promoting blood circulation, and removing blood stasis.

In our meta-analysis, about $53 \%$ of studies used Shenfu injection (SFI), which has a positive effect in supplementing Qi and warming Yang. Clinical and experimental studies have shown that SFI could act on multiple organs and prevent ischemia-reperfusion injury [7], especially for myocardial dysfunction and brain injury $[20,27]$, which may be related to its significant effect on the improvement of energy 


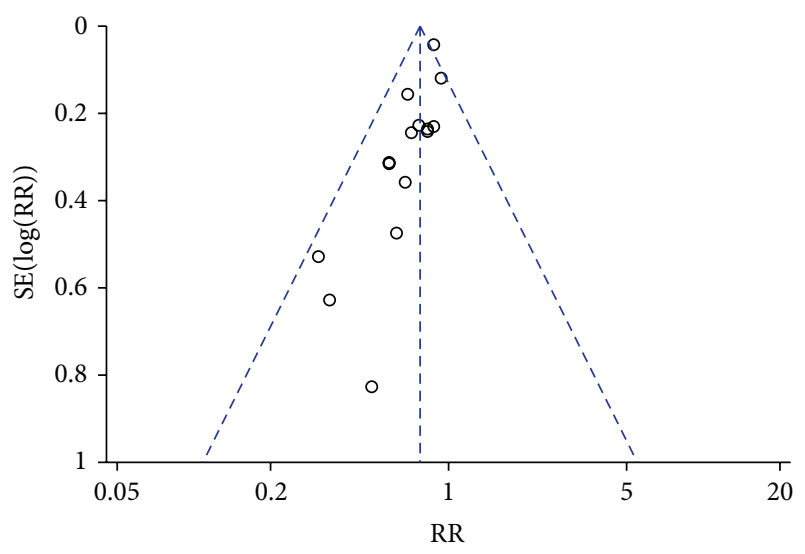

FIGURE 6: Funnel plot of all-cause mortality.

metabolism, eliminating oxygen free radical, reducing $\mathrm{Ca}^{2+}$ overload blockage, suppression of the inflammatory reaction, and inhibition of apoptosis [14, 33].

Shengmai injection (about 13\% used it in our included studies) has a perfect effect in benefiting Qi and nourishing Yin. Modern pharmacological studies have shown that it has a significant effect on strengthening the heart, boosting pressure, antiarrhythmia, and improving microcirculation $[21,28,34]$.

Another $13 \%$ of studies reported the use of Xuebijing injection, which could promote blood circulation and remove blood stasis. It has a positive effect on suppressing inflammatory response, improving hemodynamics and tissue perfusion [30]. Wu et al. reported that it could reduce the number and the extent of MODS, which directly reduce the mortality.

In summary, clinical trials have showed that combined CHM therapy has a protective effect on multiple ischemicreperfusion organs and tissues of the whole body. There were positive effects of jointing application of CHM for the treatment of CPR. Nevertheless, in consideration of the methodological quality of our included studies and the existence of bias, further studies are needed to provide more evidence to prove or refute our conclusion.

Strength and Limitations. This is the first systematic review/meta-analysis evaluating the therapeutic effect of the combined CHM therapy. Secondly, the number of participants was a relatively large sample size to clarify and confirm the issue. Thirdly, we strictly enforce the literature search, data extraction and analysis, in order to ensure the credibility of our results.

There were some limitations in our meta-analysis. Firstly, the methodological quality of the studies we included was not high. The main manifestations of the low and unclear methodological quality were as follows: for allocation sequence generation, only $26 \%$ (4/15) of the articles adequately mentioned the generation of random sequence. Allocation concealment was not described in any of the trials. Only $6 \%(1 / 15)$ of trials mentioned blinding and placebocontrolled method. All trials reported that there were no baseline differences, but only one study provided detailed information in tabular form. The number of patients at follow-up and the reasons for the failures at follow-up were not explicitly described in any of the trials. No studies involved intention-to-treat analysis (ITT). Therefore, the result of our meta-analysis should be cautiously considered due to low/unclear quality of evidence. There is hope that more clinical staff draw attention in these aspects and carry out more rigorously designed trials.

Secondly, all studies were conducted in China and published in Chinese, which indicated that there was publication bias. Meanwhile, the limitation of publication restricted the comprehension and application of CHM, especially for those readers and writers whose native language is not Chinese. The culture of Chinese medicine, as China's treasure, is extensive and profound. Therefore, we hope through more publication with foreign language to let more readers and writers recognize the benefits of combined CHM therapy and apply it.

\section{Conclusions}

This meta-analysis provides evidence suggesting that combined CHM therapy is associated with a decreased risk of mortality compared with conventional western therapy in patients after CPR. However, the findings should be interpreted cautiously in line with the overall methodological quality and potential biases of the included trials. More rigorously designed, randomized double-blind, placebocontrolled trials are necessary for further evaluating the effectiveness of combination CHM therapy.

\section{Conflict of Interests}

The authors declare no conflict of interests for publication of this paper.

\section{Authors' Contribution}

The study was conceived and designed by Xiaoguang Lu. Wenxiu Guo and Dalong Wang independently screened literature, extracted data, and evaluated study quality. Disagreements between Wenxiu Guo and Dalong Wang were determined by Xiaoguang Lu. Wenxiu Guo conducted data synthesis and analysis and wrote the paper. Xiaoguang $\mathrm{Lu}$, Dalong Wang, and Tuo Chen checked this work again. Zhiwei Fan and Yi Song participated in the discussion.

\section{Acknowledgment}

This study was supported by two funds from Natural Science Foundation of China (NSFC) (no. 81173397 and no. 81473512).

\section{References}

[1] K. Mashiko, T. Otsuka, S. Shimazaki et al., "An outcome study of out-of-hospital cardiac arrest using the Utstein template-a Japanese experience," Resuscitation, vol. 55, no. 3, pp. 241-246, 2002. 
[2] V. M. Nadkarni, G. L. Larkin, M. A. Peberdy et al., "First documented rhythm and clinical outcome from in-hospital cardiac arrest among children and adults," The Journal of the American Medical Association, vol. 295, no. 1, pp. 50-57, 2006.

[3] J. P. Nolan, J. Soar, G. B. Smith et al., "Incidence and outcome of in-hospital cardiac arrest in the United Kingdom National Cardiac Arrest Audit," Resuscitation, vol. 85, no. 8, pp. 987-992, 2014.

[4] R. W. Neumar, J. P. Nolan, C. Adrie et al., "Post-cardiac arrest syndrome: epidemiology, pathophysiology, treatment, and prognostication. A consensus statement from the International Liaison Committee on Resuscitation (American Heart Association, Australian and New Zealand Council on Resuscitation, European Resuscitation Council, Heart and Stroke Foundation of Canada, InterAmerican Heart Foundation, Resuscitation Council of Asia, and the Resuscitation Council of Southern Africa); the American Heart Association Emergency Cardiovascular Care Committee; the Council on Cardiovascular Surgery and Anesthesia; the Council on Cardiopulmonary, Perioperative, and Critical Care; the Council on Clinical Cardiology; and the Stroke Council," Circulation, vol. 118, no. 23, pp. 2452-2483, 2008.

[5] L. H. Opie, "Reperfusion injury and its pharmacologic modification," Circulation, vol. 80, no. 4, pp. 1049-1062, 1989.

[6] B. C. White, L. I. Grossman, and G. S. Krause, "Brain injury by global ischemia and reperfusion: a theoretical perspective on membrane damage and repair," Neurology, vol. 43, no. 9, pp. 1656-1665, 1993.

[7] Z. M. Shi, G. G. Guo, G. Z. Wu, F. Zhong, W. D. Song, and X. H. Zhu, "Study on the protective effects of Shengfu injection on multiple organs injury in patients after cardiopulmonary resuscitation," Journal of Clinical Emergency Call, vol. 5, no. 6, pp. 19-21, 2004.

[8] Q. R. Wang, "Clinical study of tetramethylpyrazine in patients with acute cerebral ischemia/reperfusion injury after cardiopulmonary resuscitation," China Practical Medicine, vol. 17, no. 17, p. 157, 2012.

[9] X. K. Meng, S. Q. Shi, X. Z. Zhang et al., "Applications of Jiunitongyu decoction on patients with post-cardiac arrest syndrome," The Second Youth Forum of Emergency Medicine, Chinese Journal of Emergency Medicine, pp. 197-200, 2010.

[10] L. He, C. Yang, W. T. Zhang, and X. Y. Jin, “Therapeutic efficacy of Shenfu injection in patients after cardiopulmonary resuscitation," Guide of China Medicine, vol. 10, no. 23, pp. 306307, 2012.

[11] X. D. Li, "Clinical research on treating cardiopulmonary resuscitation cerebral ischemia-reperfusion injury with TMP," Clinical Journal of Chinese Medicine, vol. 3, no. 16, pp. 79-81, 2011.

[12] H. B. Liu, Y. J. Yin, X. Q. Jiang, and J. L. Li, “Study of Xingnaojing injection on serum cytokine of patients after cardio-pulmonary resuscitation," Journal of Emergency in Traditional Chinese Medicine, vol. 18, no. 10, pp. 1617-1618, 2009.

[13] Z. J. Lou, L. M. Zhuge, W. L. Zheng, Y. K. Ou, and L. Shou, "Protective effects of tetramethylpyrazine in patients with acute cerebral ischemia/reperfusion injury after cardiopulmonary resuscitation," Chinese Journal of Integrated Traditional and Western Medicine in Intensive and Critical Care, vol. 10, no. 5, pp. 299-301, 2003.

[14] Z.-F. Wang, L. Zhong, and Y.-S. Li, "The protective effects of Shenfu injection on the global cerebral ischemia/reperfusion injury of rats," Zhongguo Ying Yong Sheng Li Xue Za Zhi, vol. 28, no. 5, pp. 462-465, 2014.

[15] D. Moher, L. Shamseer, M. Clarke et al., "Preferred reporting items for systematic review and meta-analysis protocols (PRISMA-P) 2015 statement," Systematic Reviews, vol. 4, no. 1, article 1, 2015.

[16] J. P. T. Higgins and S. Green, "Review: cochrane handbook for systematic reviews for interventions, Version 5.1.0, published 3/2011. Julian P.T. Higgins and Sally Green, Editors," Research Synthesis Methods, vol. 2, no. 2, pp. 126-130, 2011.

[17] J. Wetterslev, K. Thorlund, J. Brok, and C. Gluud, "Estimating required information size by quantifying diversity in randomeffects model meta-analyses," BMC Medical Research Methodology, vol. 9, article 86, 2009.

[18] S. R. Sun, C. L. Ma, and X. Y. Mo, "Clinical observation of Erhuang powder in patients with gastrointestinal dysfunction after cardiopulmonary resuscitation," Journal of New Chinese Medicine, no. 10, pp. 45-46, 2009.

[19] Y. He, X. R. Xie, and Q. Chen, "Analysis on the effect of Shenfu injection on post-cardiac arrest syndrome," National Medical Frontiers of China, vol. 8, no. 15, p. 33, 2013.

[20] J. L. Hu, F. J. Li, C. R. Hu, and C. Y. Zhang, "Protective effect of Shenfu injection on myocardium during cardiopulmonary resuscitation," Chinese Journal of cardiovascular Rehabilitation Medicine, vol. 18, no. 1, pp. 73-75, 2009.

[21] M. Q. Yi, X. F. Wang, and Y. M. Zhang, "Protective effects of Shengmai injection in elderly patients with myocardial ischemia/reperfusion injury after cardiopulmonary resuscitation," Chinese Journal of Gerontology, vol. 32, no. 24, pp. 53855386, 2012.

[22] S. Y. Xu, X. L. Li, J. Chen, and C. T. Feng, "Study on the application of Shenfu injection in patients with cardiac arrest treated with cardiopulmonary resuscitation," Modern Journal of Integrated Traditional Chinese and Western Medicine, vol. 17, no. 16, pp. 2433-2434, 2008.

[23] J. Su, J. Z. Liang, and B. Zhang, "Clinical exploration on Shenfu injection on post-cardiac arrest syndrome," China Journal of Emergency Resuscitation and Disaster Medicine, vol. 6, no. 10, pp. 879-881, 2011.

[24] G. Y. Hu, "Effects of combination Shengfu injection with conventional methods for CPR," Chinese Journal of Experimental Traditional Medical Formulae, vol. 18, no. 12, pp. 293-295, 2012.

[25] Q. Y. Deng, L. T. Tao, and D. W. Wang, "Clinical studies of Shenfu Injection in patients for cardiopulmonary resuscitation," Journal of New Chinese Medicine, vol. 44, no. 4, pp. 15-17, 2012.

[26] X. J. Zhang and F. Y. Sun, "Effects of ginseng-monkshood extract on patients with post-cardiac arrest syndrome," Gansu Medical Journal, vol. 31, no. 2, pp. 106-108, 2012.

[27] H. X. Liu, "Protective effects of Shengfu injection in patients with myocardial injury after cardiopulmonary resuscitation," China Practical Medicine, vol. 8, no. 17, pp. 164-165, 2013.

[28] S. J. Zhao, Y. S. Wang, Z. M. Liu, and A. Y. Qu, "Protective effect of Shengmai injection on myocardium during the cardiopulmonary resuscitation," Chinese Journal of Critical Care Medicine, vol. 27, no. 4, pp. 292-294, 2007.

[29] X. D. Wu, J. Z. Zhang, X. Y. Wan, and R. H. Huang, "Preventive and therapeutic effects of Xuebijing on post-resuscitation syndrome:analysis of 41 cases," China Journal of Emergency Resuscitation and Disaster Medicine, no. 4, pp. 251-252, 2009.

[30] D. Y. Liang, C. L. Ma, J. M. Huang, S. C. Mo, Z. J. Lin, and H. Lin, "Effect of Xuebijing injection on haemodynamics and tissue 
perfusion of the patients after cardiopulmonary resuscitation," Medical Journal of West China, vol. 26, no. 3, pp. 296-297, 300, 2014.

[31] D. Y. Liang, C. L. Ma, Z. J. Lin, S. C. Mo, and J. M. Huang, "Observation on the effect of Sini decoction on treatment of cardiac dysfunction after cardiopulmonary resuscitation," Journal of Guangxi Traditional Chinese Medical University, vol. 16, no. 2, pp. 32-34, 2013.

[32] K. M. Li and W. P. Liu, "Therapeutic effects of Xuefuzhuyu decoction on post-resuscitation syndrome: analysis of 20 cases," Henan Traditional Chinese Medicine, vol. 34, no. 3, pp. 416-417, 2014.

[33] S.-Y. Zheng, J. Sun, X. Zhao, and J.-G. Xu, "Protective effect of Shen-Fu on myocardial ischemia-reperfusion injury in rats," The American Journal of Chinese Medicine, vol. 32, no. 2, pp. 209-220, 2004.

[34] L. Yin and X. Wo, "Pharmacological and clinical research progress of Shenmai injection," Journal of Zhejiang College of Traditional Chinese Medicine, vol. 25, no. 6, pp. 65-68, 2001. 


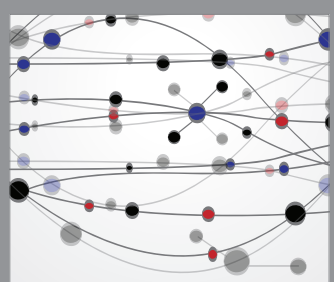

The Scientific World Journal
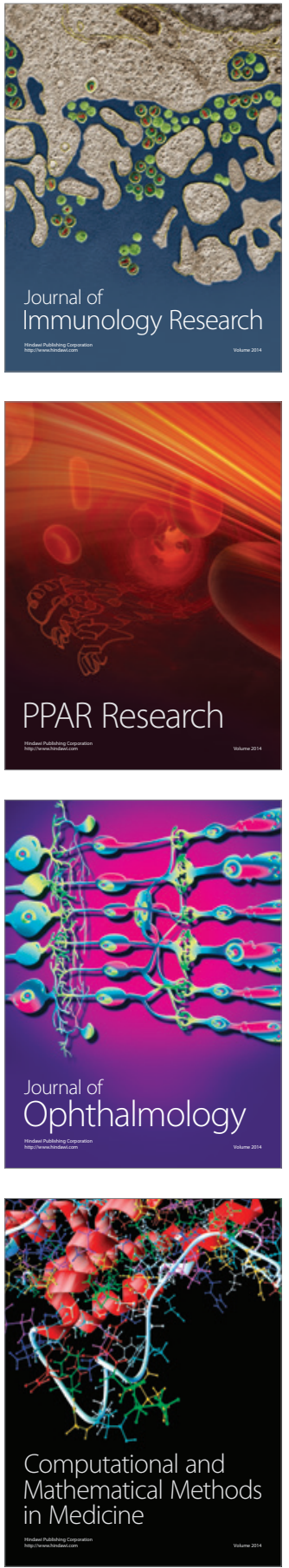

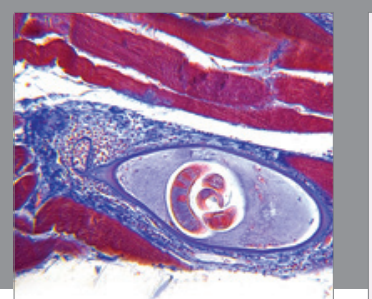

Gastroenterology Research and Practice

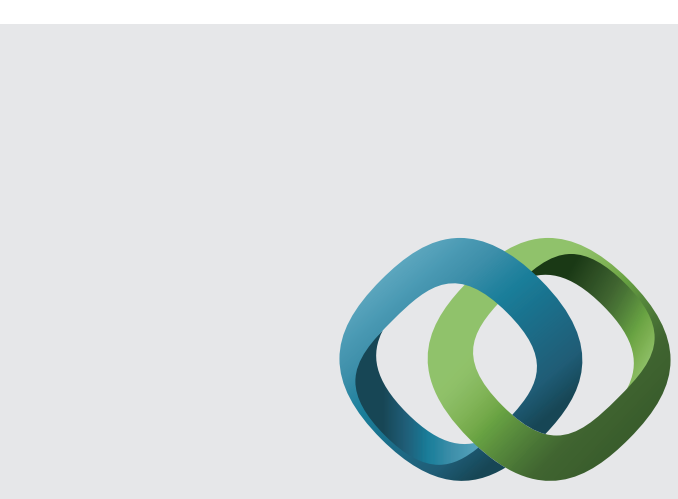

\section{Hindawi}

Submit your manuscripts at

http://www.hindawi.com
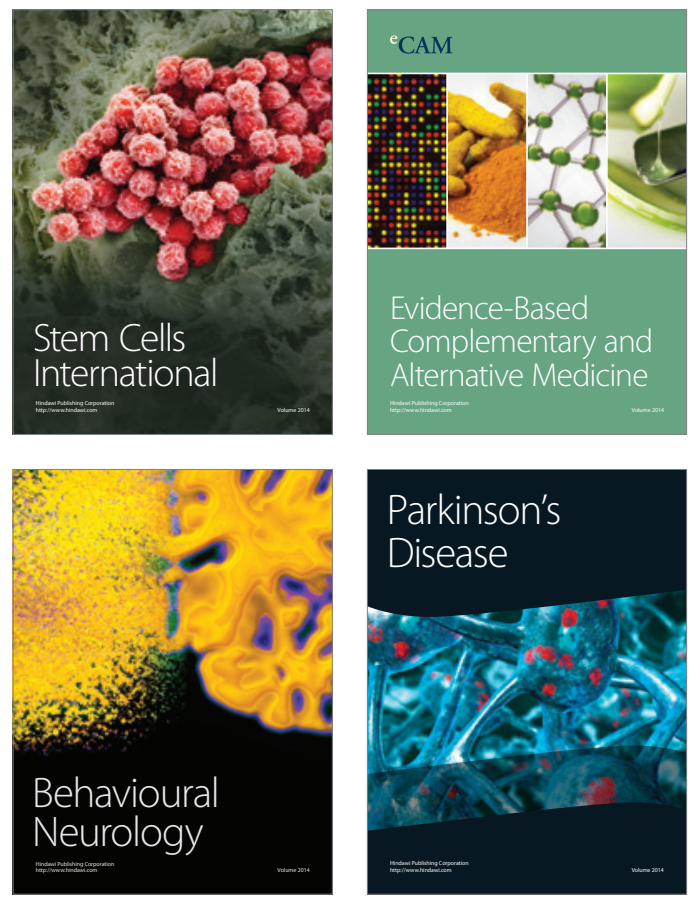
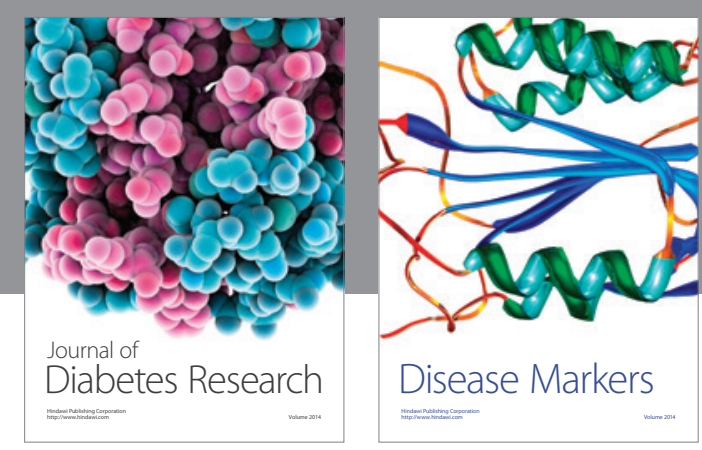

Disease Markers
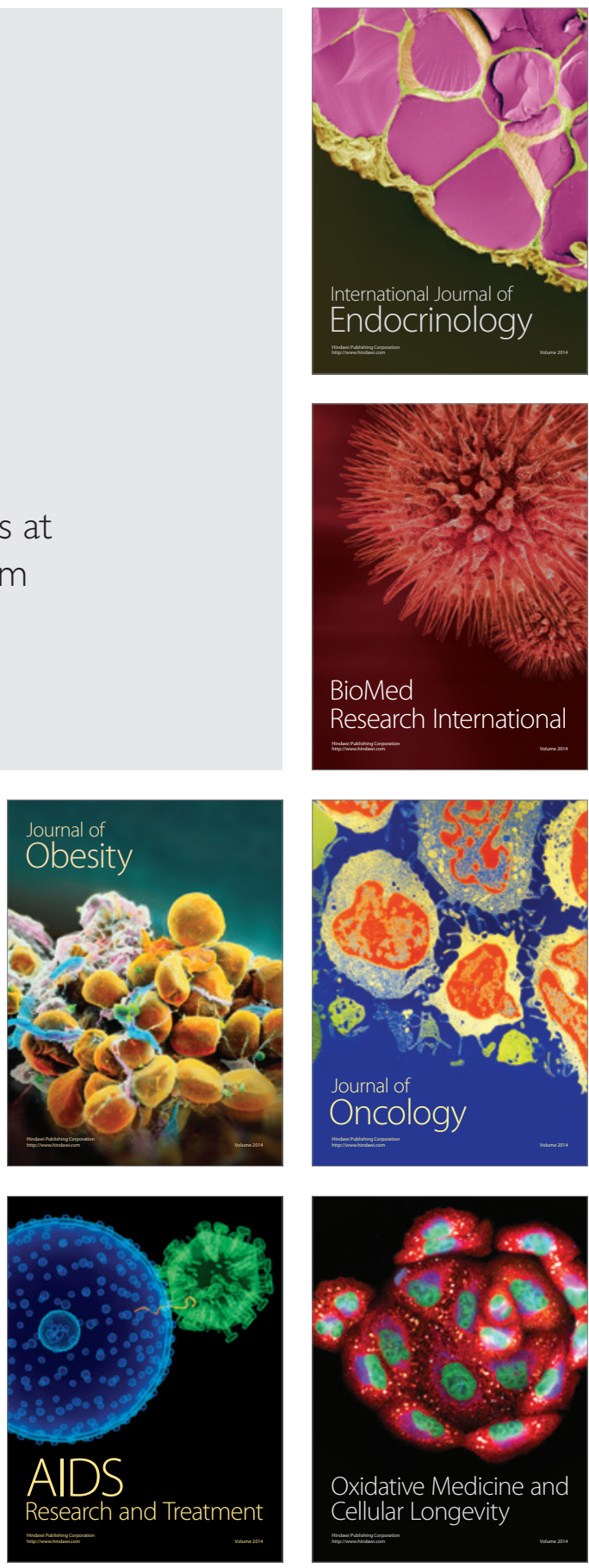